\title{
Atrial Natriuretic Peptide Protects against Histamine-Induced Endothelial Barrier Dysfunction in Vivo $\$$
}

\author{
Robert Fürst, Martin F. Bubik, Peter Bihari, Bettina A. Mayer, Alexander G. Khandoga, \\ Florian Hoffmann, Markus Rehberg, Fritz Krombach, Stefan Zahler, and \\ Angelika M. Vollmar \\ Department of Pharmacy, Pharmaceutical Biology (R.F., M.F.B., B.A.M., F.H., S.Z., A.M.V.) and Institute for Surgical Research \\ (P.B., A.G.K., M.R., F.K.), University of Munich, Germany
}

Received January 30, 2008; accepted April 11, 2008

\begin{abstract}
Endothelial barrier dysfunction is a hallmark of many severe pathologies, including sepsis or atherosclerosis. The cardiovascular hormone atrial natriuretic peptide (ANP) has increasingly been suggested to counteract endothelial leakage. Surprisingly, the precise in vivo relevance of these observations has never been evaluated. Thus, we aimed to clarify this issue and, moreover, to identify the permeability-controlling subcellular systems that are targeted by ANP. Histamine was used as important pro-inflammatory, permeability-increasing stimulus. Measurements of fluorescein isothiocyanate (FITC)-dextran extravasation from venules of the mouse cremaster muscle and rat hematocrit values were performed to judge changes of endothelial permeability in vivo. It is noteworthy that ANP strongly reduced the histamine-evoked endothelial barrier dys-
\end{abstract}

function in vivo. In vitro, ANP blocked the breakdown of transendothelial electrical resistance (TEER) induced by histamine. Moreover, as judged by immunocytochemistry and Western blot analysis, ANP inhibited changes of vascular endothelial (VE)-cadherin, $\beta$-catenin, and $\mathrm{p} 120^{\mathrm{ctn}}$ morphology; VE-cadherin and myosin light chain 2 (MLC2) phosphorylation; and F-actin stress fiber formation. These changes seem to be predominantly mediated by the natriuretic peptide receptor (NPR)-A, but not by NPR-C. In summary, we revealed ANP as a potent endothelial barrier protecting agent in vivo and identified adherens junctions and the contractile apparatus as subcellular systems targeted by ANP. Thus, our study highlights ANP as an interesting pharmacological compound opening new therapeutic options for preventing endothelial leakage.
The endothelium crucially participates in the regulation of important physiological functions, such as blood pressure, coagulation, or host defense, and it represents a barrier that controls the passage of cells, macromolecules, and fluid between the blood and the adjacent tissue interstitium. Beyond its physiological role, the endothelium is also involved in pathological conditions: endothelial barrier dysfunction is a hallmark of inflammatory processes and still poses an important therapeutical challenge, because a causal pharmacological treatment remains widely lacking.

Article, publication date, and citation information can be found at http://molpharm.aspetjournals.org.

doi:10.1124/mol.108.045773.

S The online version of this article (available at http://molpharm. aspetjournals.org) contains supplemental material.
Endothelial barrier function is mainly governed by the balance between interendothelial cell adhesion and retraction. Adherens junctions (AJs) are important subcellular structures responsible for endothelial cell-cell attachment, and they represent multiprotein complexes that consist of vascular endothelial (VE)-cadherin, $\beta$-catenin, and $\mathrm{p} 120^{\text {ctn }}$. Under inflammatory conditions, VE-cadherin junctions disassemble, thus facilitating paracellular passage, and show an increased tyrosine phosphorylation. Endothelial cell retraction is caused by the activation of the contractile machinery [i.e., the interaction between actin and myosin, which is controlled by phosphorylation of the myosin light chain (MLC)]. These two regulatory systems could be targets of a successful therapeutic principle. 
The cardiovascular hormone atrial natriuretic peptide (ANP) is secreted by the cardiac atria as response to an increased plasma volume. In general, ANP binds to the guanylate cyclase-coupled natriuretic peptide receptor (NPR)-A and NPR-C, which lacks guanylate cyclase function. ANP exerts a hypotensive effect by its natriuretic, diuretic, and vasodilating action. The role of ANP as an important regulator of the cardiovascular system is highlighted by the fact that ANP [carperitide (HANP)] has been approved as drug for the treatment of acute heart failure in Japan. However, ANP has been recognized to possess important additional functions beyond blood pressure regulation: ANP is expressed by macrophages and is able to influence these immune cells by attenuating their inflammatory response (Kiemer and Vollmar, 2001). Most importantly, ANP exerts anti-inflammatory properties in the endothelium (Kiemer et al., 2005). Thus, we posed the working hypothesis that ANP could open new therapeutical options for protecting against endothelial barrier dysfunction. In fact, some evidence is given from in vitro and ex vivo experiments that ANP influences an inflammation-increased permeability (Inomata et al., 1987; Lofton et al., 1991; Kiemer et al., 2002a). However, data precisely demonstrating a beneficial effect of administered ANP on inflammation-induced endothelial barrier dysfunction in vivo are lacking. Moreover, data concerning the effect of ANP on subcellular systems that control permeability are missing.

Therefore, the aims of the study were 1) to examine the in vivo potential of ANP as pharmacological agent counteracting endothelial leakage and 2) to investigate the influence of ANP on key regulators of endothelial permeability [i.e., the endothelial cell adhesion (VE-cadherin) and contraction system (MLC)].

\section{Materials and Methods}

Measurement of Vascular Permeability in the Mouse Cremaster Muscle in Vivo. Male C57BL/6NCrl mice (Charles River Laboratories, Sulzfeld, Germany) weighing 23 to $25 \mathrm{~g}$ were used. All experiments were performed according to the German legislation for the protection of animals. Surgery was performed as described by Baez (1973). Vascular permeability was analyzed according to Hatekeyama et al. (2006). In brief, mice were anesthetized i.p. using a ketamine (Pfizer, Karlsruhe, Germany)/xylazine (Bayer, Leverkusen, Germany) mixture. Fluorescein isothiocyanate-dextran (150 kDa; Sigma-Aldrich, Taufkirchen, Germany), Ringer solution (control), and ANP (bolus sufficient to reach $200 \mathrm{nM}$ plasma concentration; AnaSpec/MoBiTec, Göttingen, Germany) were applied into the left femoral artery. Twenty minutes after ANP application, the cremaster was superfused with histamine (30 $\mu \mathrm{M}$; Sigma-Aldrich) for $10 \mathrm{~min}$. Dexamethasone 21-phosphate (disodium salt; SigmaAldrich) was administered i.p. (10 mg/kg of body weight) $2 \mathrm{~h}$ before histamine. Postcapillary venules with diameters of 18 to $30 \mu \mathrm{m}$ were analyzed. Ten regions of interests $\left(50 \times 50 \mu \mathrm{m}^{2}\right)$ in the interstitial tissue (approximately $50 \mu \mathrm{m}$ distant from the venule) were randomly selected. Intravital microscopic images were recorded with an IMAGO charge-coupled device camera (TILL Photonics, Gräfelfing, Germany) and subjected to digital image analysis (TILLvisION 4.0; TILL Photonics).

Measurement of Rat Hematocrit. Male Sprague-Dawley rats (Charles River Laboratories) weighing 190 to $240 \mathrm{~g}$ were used. All experiments were performed according to the German legislation for the protection of animals. Rats were anesthetized i.p. using a fentanyl (Jansen-Cilag, Neuss, Germany)/midazolam (Ratiopharm, Ulm,
Germany) mixture, and anesthesia was maintained by $1.5 \%$ isoflurane (Abbott, Wiesbaden, Germany). Rats were pretreated for $15 \mathrm{~min}$ with ANP (bolus sufficient to reach $200 \mathrm{nM}$ plasma concentration) or PBS, followed by histamine (bolus sufficient to reach $1 \mu \mathrm{M}$ plasma concentration). Reagents were applied into the jugular vein. Thirty minutes after administration of histamine, blood samples were collected via a jugular artery catheter, and hematocrit was determined by centrifugation in hematocrit capillaries.

Cell Culture. Human umbilical vein endothelial cells (HUVECs) were prepared as described previously (Kiemer et al., 2002a) and cultured in endothelial cell growth medium (Provitro, Berlin, Germany) containing $10 \%$ fetal bovine serum (Biochrom, Berlin, Germany). Cells were used for experiments at passages 1 to 3 .

Measurement of Transendothelial Electric Resistance (TEER). HUVECs were cultured on collagen A (Biochrom)-coated Millicell 12-mm PCF inserts (Millipore, Schwalbach, Germany). TEER measurements were performed with an Ussing-type chamber. The incubation fluid (HEPES-buffer containing $10 \%$ fetal bovine serum) was circulated by means of humidified air streams at $37^{\circ} \mathrm{C}$. A custom-built voltage/current clamp unit in connection with a computer-aided evaluation program was used. Bidirectional square current pulses of $50 \mu \mathrm{A}$ and $200 \mathrm{~ms}$ duration were applied across the monolayer every $2 \mathrm{~s}$. The resistance of the monolayer was calculated by Ohm's law from the induced deflection of the transendothelial voltage.

Immunocytochemistry/Histochemistry and Confocal Laser Scanning Fluorescence Microscopy. HUVECs were cultured on collagen-treated $\mu$-Slides (ibidi, Martinsried, Germany). The NPR-A/B antagonist HS-142-1 (Morishita et al., 1991) was kindly provided by Dr. Y. Matsuda (Kyowa Hakko Kogyo Co., Ltd., Shizuoka, Japan). cANP was from Bachem (Weil am Rhein, Germany). HUVECs and samples of the mouse cremaster muscle (immediately dissected after histamine treatment) were analyzed immunocytochemically/histochemically and by confocal fluorescence microscopy as described previously (Fürst et al., 2005). The following antibodies and reagents were used: mouse monoclonal anti-VE-cadherin (Santa Cruz, Heidelberg, Germany), rabbit polyclonal anti-phospho-Tyr ${ }^{731}$-VE-cadherin (Biosource/Invitrogen, Karlsruhe, Germany), rabbit polyclonal anti-phospho-MLC2 ( $\mathrm{Thr}^{18}$ / Ser ${ }^{19}$; Cell Signaling/New England Biolabs, Frankfurt am Main, Germany), rhodamine phalloidin (Invitrogen, Karlsruhe, Germany), Alexa Fluor 633-linked goat anti-mouse (Invitrogen), and Alexa Fluor 488linked goat anti-rabbit (Invitrogen).

Western Blot Analysis. HUVECs were cultured in collagentreated six-well plates or $60-\mathrm{mm}$ dishes. Western blot analysis was performed as described previously (Kiemer et al., 2002a). The following antibodies were used: rabbit polyclonal anti-phospho-Tyr ${ }^{731}$ VE-cadherin (BioSource International, Camarillo, CA), mouse monoclonal anti-VE-cadherin (Santa Cruz), rabbit polyclonal anitphospho-MLC2 ( $\mathrm{Thr}^{18} / \mathrm{Ser}^{19}$ ) (Cell Signaling), and MLC2 (Santa Cruz).

Statistical Analysis. Statistical analysis was performed with Prism software (version 3.03; GraphPad Software, San Diego, CA). Unpaired $t$ test was used to compare two groups. To compare three or more groups, one-way analysis of variance followed by NewmanKeuls post hoc test was used.

\section{Results}

ANP Protection against an Inflammation-Impaired Endothelial Barrier Function in Vivo. To judge endothelial permeability in vivo, we measured the extravasation of FITC-dextran $(150 \mathrm{kDa})$ via intravital fluorescence microscopy in postcapillary venules of the mouse cremaster muscle. Twenty minutes after intra-arterial application of ANP (bolus sufficient to reach $200 \mathrm{nM}$ plasma concentration), histamine $(30 \mu \mathrm{M})$ was superfused for $10 \mathrm{~min}$. Histamine evoked a strong leak of FITC-dextran from the blood into the adja- 
A
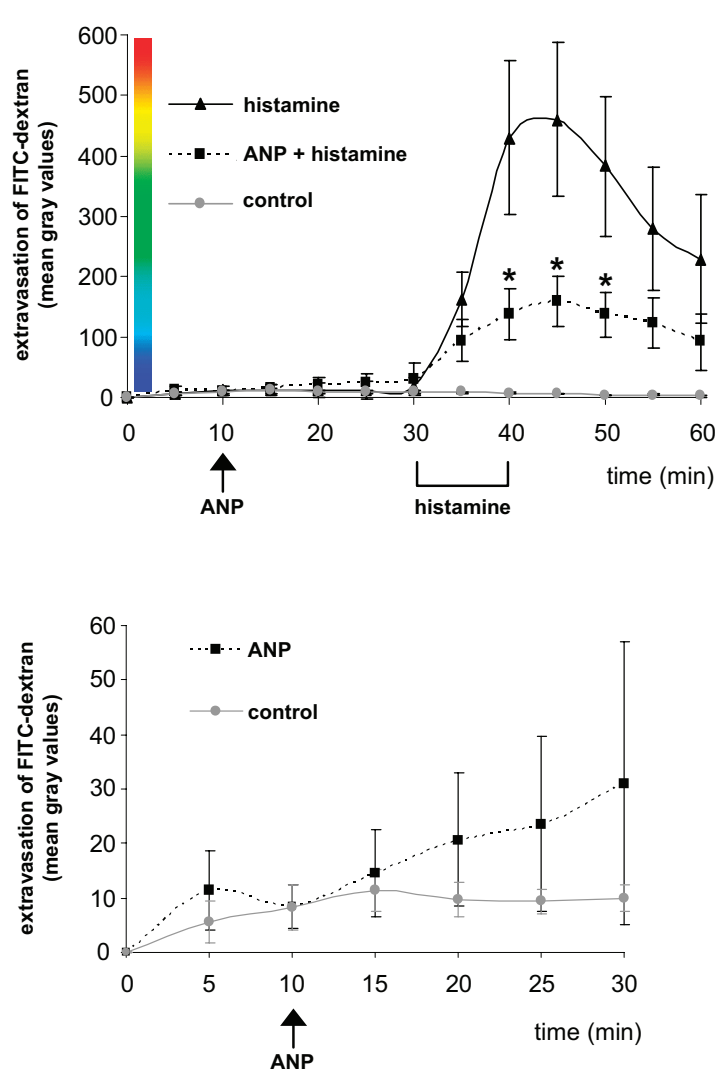

B

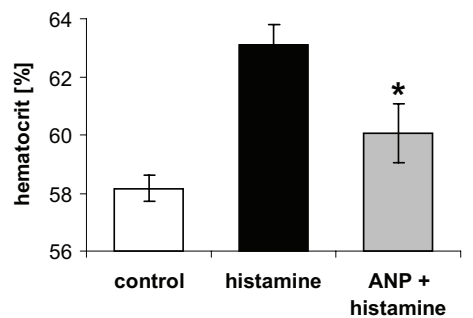

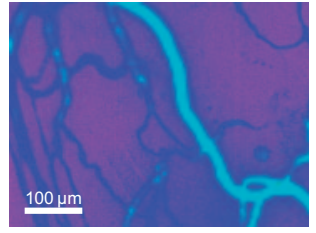

control

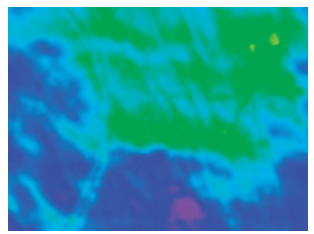

ANP + histamine

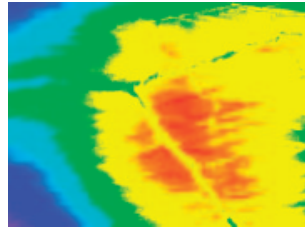

histamine

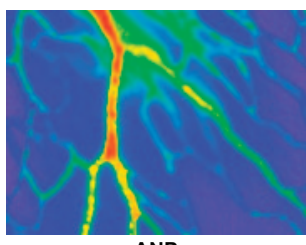

ANP

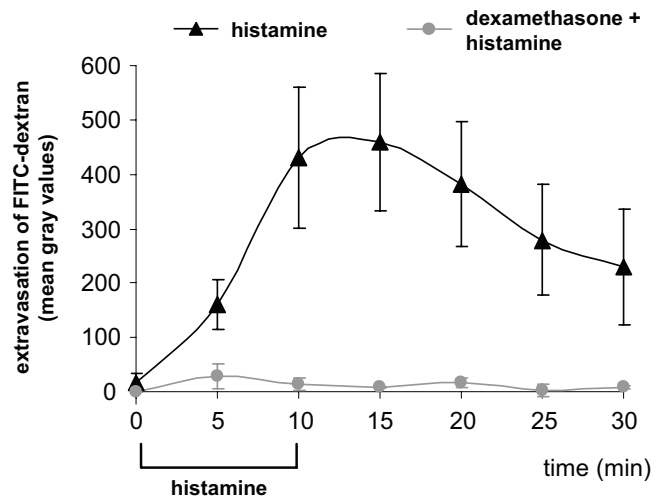

Fig. 1. ANP attenuated histamine-induced increase of endothelial permeability in vivo and in vitro. A, extravasation of FITC-dextran (150 kDa) from venules of the mouse cremaster muscle was measured. Top left, mice were pretreated with Ringer's solution (control and histamine group) or with ANP (intra-arterial bolus injection sufficient to reach $200 \mathrm{nM}$ plasma concentration). After 20 min, histamine ( $30 \mu \mathrm{M}$ ) was superfused for 10 min (histamine and ANP+histamine group). Data are expressed as mean \pm S.E.M. $(n=6) . *, p \leq 0.05$ versus histamine. Top right, one representative image is shown for each group of treatment (at time point $45 \mathrm{~min}$ for control, histamine, and histamine + ANP; at time point 30 min for ANP alone). Videos showing this FITC-dextran extravasation (movie1, control; movie2, histamine; movie3, ANP+histamine) are available as supplemental data. Bottom left, mice were treated with Ringer solution (control group) or with ANP (intra-arterial bolus injection sufficient to reach $200 \mathrm{nM}$ plasma concentration). Data are expressed as mean \pm S.E.M. $(n=6)$. Bottom right, mice were pretreated with Ringer's solution (histamine group) or with dexamethasone (10 $\mathrm{mg} / \mathrm{kg}$ of body weight, i.p.) for $2 \mathrm{~h}$. Histamine $(30 \mu \mathrm{M})$ was superfused for $10 \mathrm{~min}$. Data are expressed as mean \pm S.E.M. $(n=2)$. B, plasma volume changes were determined by measuring hematocrit values. Rats were pretreated with PBS (control) or with ANP (i.v. bolus injection sufficient to reach $200 \mathrm{nM}$ plasma concentration). After $15 \mathrm{~min}$, histamine was applied (i.v. bolus injection sufficient to reach $1 \mu \mathrm{M}$ plasma concentration). 30 min later, blood samples were taken and hematocrit was measured. Data are expressed as mean \pm S.E.M. $(n=3)$. $*, p \leq 0.05$ versus histamine. C, transendothelial electrical resistance (TEER) was used to judge changes in endothelial permeability of HUVECs. Left, histamine concentrationdependently decreases TEER values. Middle, ANP $(1 \mu \mathrm{M}, 30$ min pretreatment) attenuates the histamine-induced decrease of electrical resistance. One representative graph of four independent experiments is shown. Right, statistical analysis of all experiments performed $(n=4)$. Data are expressed as mean \pm S.E.M. $*, p \leq 0.05$ versus histamine. 
cent tissue. ANP clearly abrogated the histamine-induced extravasation (Fig. 1A, top). Movies of this extravasation are presented as supplemental data (movie1, control; movie2, histamine; movie3, ANP + histamine). ANP alone (at least in the observed 20-min pretreatment) seemed to slightly increase basal permeability (please note the different ordinate scales in Fig. 1A), but this effect was statistically not significant (Fig. 1A, bottom left). Moreover, we aimed to appraise the therapeutical impact of ANP by comparing its beneficial effect with that of a strong anti-inflammatory drug. Thus, we treated mice with a high dose of dexamethasone $(10 \mathrm{mg} / \mathrm{kg}$ i.p., $2 \mathrm{~h}$ ) before applying histamine $(30 \mu \mathrm{M})$. The glucocorticoid completely prevented the histamine-induced extravasation of FITC-dextran.

As a second approach for detecting changes of endothelial permeability in vivo, we measured hematocrit levels. Rats were treated with histamine (i.v. bolus sufficient to reach 1 $\mu \mathrm{M}$ plasma concentration), and hematocrit concentration was determined after $30 \mathrm{~min}$. Because of a reduction in plasma volume (i.e., augmented fluid extravasation), histamine evoked a strong hematocrit increase. ANP (bolus injection sufficient to reach $200 \mathrm{nM}$ plasma concentration, 15-min pretreatment) significantly reduced the permeability-increasing effect of histamine (Fig. 1B).

Characterization of the Barrier-Protecting Effect of ANP in Vitro. Data about an influence of ANP on histamineinduced endothelial leakage in vitro are completely lacking. Thus, we first aimed to verify the effect of ANP in HUVECs. To judge permeability changes, TEER was measured. Upon applying histamine, the electrical resistance of a HUVEC monolayer rapidly dropped within seconds and recovered after approximately $10 \mathrm{~min}$. The extent of this effect is dependent on the histamine concentration used: the resistance was lowered to $55 \%$ by $10 \mu \mathrm{M}$ and to $85 \%$ by $1 \mu \mathrm{M}$ histamine (Fig. 1C, left). ANP (1 $\mu \mathrm{M}, 30$ min pretreatment) attenuated the drop-down of electrical resistance evoked by histamine (Fig. 1C, middle). The statistical analysis of all experiments $(n=4)$ performed is depicted in Fig. 1C, right. The large
A

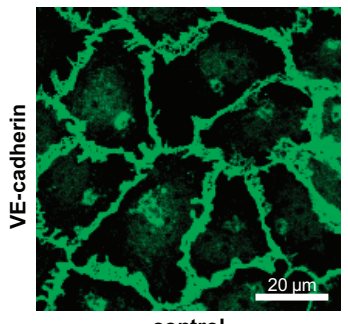

control

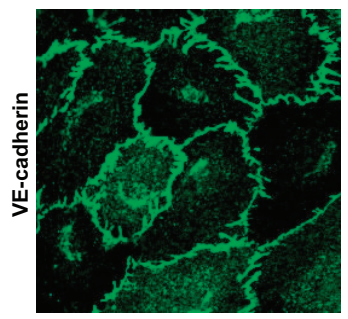

HS-142-1 + ANP + histamine

B

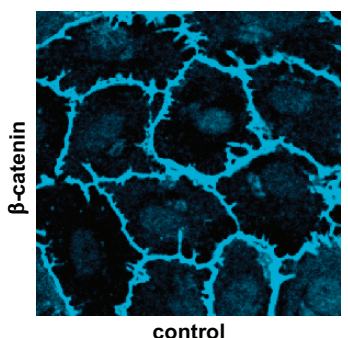

control

C

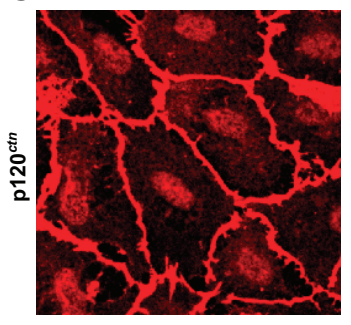

control

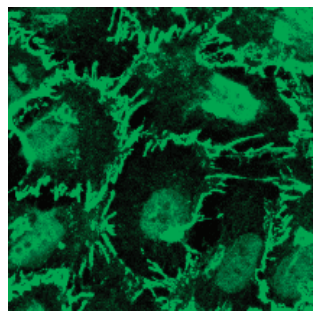

histamine

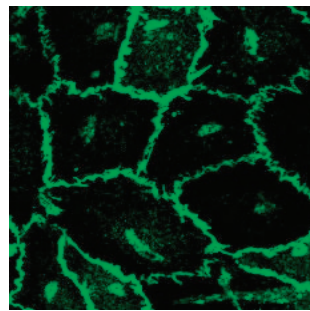

HS-142-1

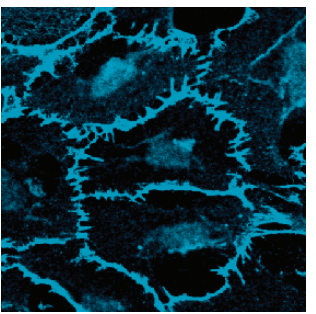

histamine

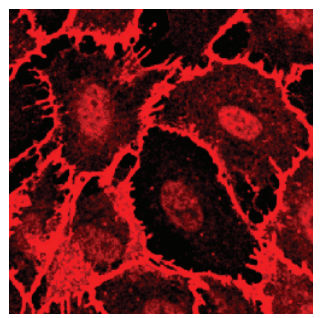

histamine

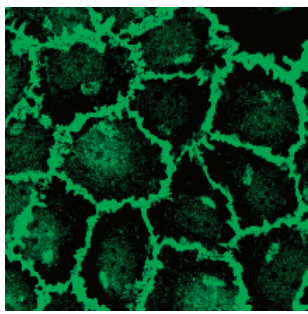

ANP + histamine

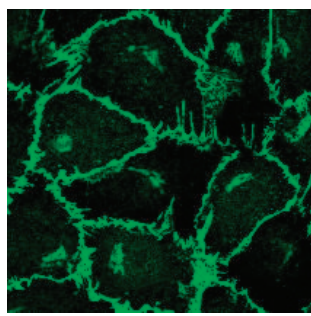

cANF + histamine

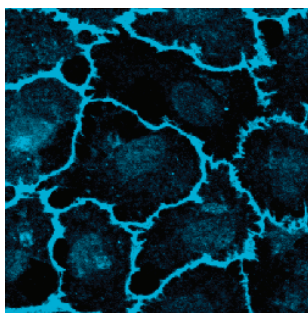

ANP + histamine

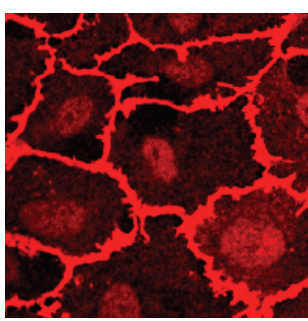

ANP + histamine

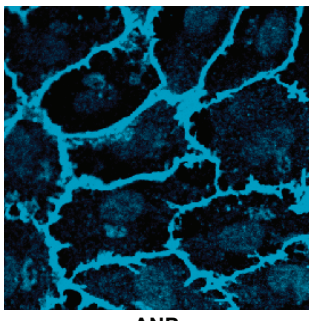

ANP

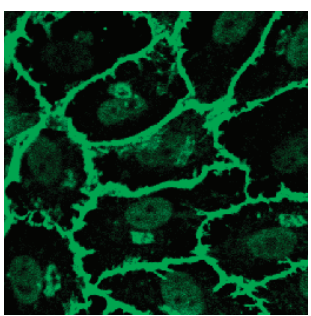

ANP

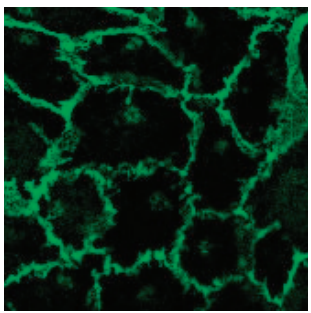

cANF

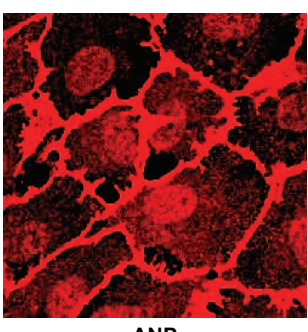

Fig. 2. ANP inhibited the histamineevoked morphological changes of adherens junctions. HUVECs were left untreated (control) or were treated with histamine (1 $\mu \mathrm{M}, 2 \mathrm{~min})$ alone or in combination with ANP $(1 \mu \mathrm{M}, 30 \mathrm{~min}$ pretreatment) or the NPR-C agonist cANF (1 $\mu \mathrm{M}, 30$ min pretreatment) The NPR-A/B receptor antagonist HS$142-1(10 \mu \mathrm{g} / \mathrm{ml})$ was given $10 \mathrm{~min}$ before ANP. Immunocytochemistry and confocal fluorescence microscopy were performed to analyze morphological changes of VE-cadherin (A), $\beta$-catenin (B), and $\mathrm{p} 120^{c t n}(\mathrm{C})$. One representative image of three independent experiments is shown. 
variability of the ANP + histamine group expresses the fact that in two of the four experiments, ANP not only attenuated the effect of histamine but also increased the endothelial resistance (i.e., led to a less permeable endothelium), even if compared with the basal resistance under control conditions. In summary, ANP strongly alleviated endothelial barrier dysfunction induced by histamine in vitro. This warranted the usage of this system for the following investigations into the action of ANP on adherens junctions and the contractile machinery.

ANP Effects on the Histamine-Evoked Changes of Adherens Junction Morphology and the HistamineInduced VE-Cadherin Tyrosine Phosphorylation. Histamine $(1 \mu \mathrm{M})$ led to strong changes of AJ morphology: the VE-cadherin, $\beta$-catenin, and $\mathrm{p} 120^{\text {ctn }}$ seam, properly built in untreated endothelial cells (control), becomes fringy, indicating an AJ disassembly (i.e., the retraction of the interendothelial VE-cadherin homodimers and/or an intramembranous lateral shift) (Fig. 2, A-C). Endothelial cells treated with ANP alone showed no effect on AJs. Most importantly, ANP (1 $\mu \mathrm{M}$, 30-min pretreatment) clearly abolished the detrimental effects induced by histamine (Fig. 2, A-C).

To clarify which natriuretic peptide receptor is involved in mediating the beneficial actions of ANP, we treated cells with the NPR-A/B antagonist HS-142-1 (10 $\mu \mathrm{g} / \mathrm{ml}, 10 \mathrm{~min}$ before ANP) and found that the effects on VE-cadherin disassembly were prevented by this inhibitor. The NPR-C receptor agonist cANF (1 $\mu \mathrm{M}, 30 \mathrm{~min}$ before histamine) was unable to mimic the effects of ANP (Fig. 2A). Compared with NPR-B, NPR-A binds ANP with a much higher affinity. Thus, our results suggest that the action of ANP was mainly transduced by NPR-A. The C-receptor seemed not to be involved.
Phosphorylation of the VE-cadherin $\mathrm{Tyr}^{731}$ residue is associated with AJ disassembly and strong endothelial leakage in vitro (Potter et al., 2005). First, we verified that $\mathrm{Tyr}^{731}$ was also phosphorylated by histamine in vivo: vessels of the mouse cremaster muscle showed a strong increase of $\mathrm{Tyr}^{731}$ phosphorylation induced by histamine (30 $\mu \mathrm{M}, 10 \mathrm{~min}$; Fig. $3 \mathrm{~A})$ and the same pronounced localization at cell fringes (Fig. $3 \mathrm{~A}$, longitudinal vessel section) as in the in vitro situation (Fig. 3B). Most importantly, as shown both by microscopic (Fig. 3B) and by Western blot analysis (Fig. 3C), ANP completely blocked the histamine-induced VE-cadherin $\mathrm{Tyr}^{731}$ phosphorylation. ANP alone evoked no alterations of the phosphorylation (Fig. 2B). Our data clearly point toward a protecting effect of ANP on the integrity of endothelial adherens junctions.

ANP Effects on the Histamine-Evoked Activation of MLC and the Formation of F-Actin Stress Fibers. The generation of contractile forces (interaction of actin and myosin) is governed by MLC $\mathrm{Thr}^{18} / \mathrm{Ser}^{19}$-phosphorylation. Histamine treatment time-dependently led to a strong phosphorylation of MLC, which was analyzed microscopically (Fig. 4A) and by Western blotting (Fig. 4B). Moreover, histamine evoked a strong change in F-actin organization. Although quiescent endothelial cells showed a cortical F-actin localization, histamine induced the formation of long, cell-spanning stress fibers (Fig. 4C). ANP clearly reduced both MLC phosphorylation (Fig. 4, A and B) and F-actin stress fiber formation (Fig. 4C). ANP alone had no effect on these parameters (Fig. 4, A-C). These results indicate that ANP prevented histamine-evoked activation of the endothelial cell contraction system.

Furthermore, we investigated which NP receptor subtype

A

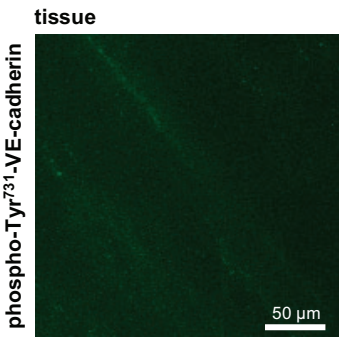

control

B

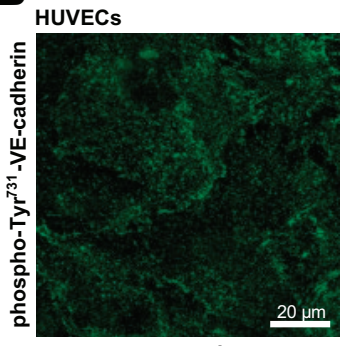

control
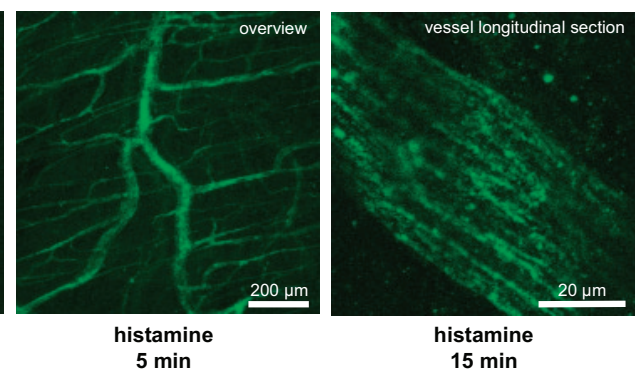

15 min

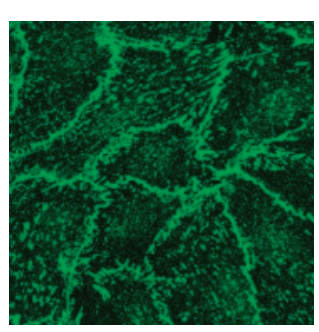

histamine

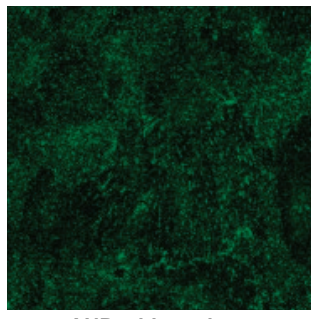

ANP + histamine

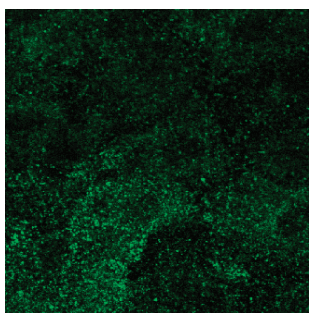

ANP

Fig. 3. ANP blocked histamine-induced VE-cadherin Tyr ${ }^{731}$-phosphorylation. A, histamine induces phosphorylation of VE-cadherin at $\mathrm{Tyr}^{731}$ in vivo. Mice were treated as described in Fig. 1A. Samples of the mouse cremaster muscle were analyzed via immunohistochemistry and confocal fluorescence microscopy. Histamine (30 $\mu \mathrm{M})$ was superfused for the indicated times. One representative image of three independent experiments is shown. B and C, ANP inhibits histamine-induced VE-cadherin $\mathrm{Tyr}^{731}$-phosphorylation in vitro. HUVECs were left untreated (control) or were treated with histamine $(1 \mu \mathrm{M}, 5 \mathrm{~min})$ or $\operatorname{ANP}(1 \mu \mathrm{M}$, $30 \mathrm{~min})$ alone or with ANP $(1 \mu \mathrm{M}) 30$ min before histamine was applied. The VE-cadherin $\mathrm{Tyr}^{731}$-phosphorylation was analyzed via immunocytochemistry and confocal fluorescence microscopy $(\mathrm{B}, n=3)$ or biochemically via Western blot $(\mathrm{C}, n=2)$.

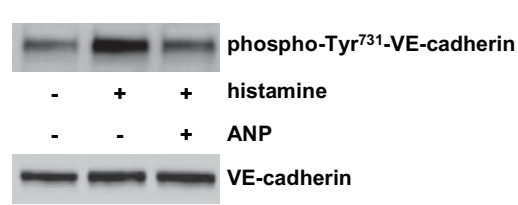


was involved in mediating these effects. The NPR-A/B inhibitor HS-142-1 $(10 \mu \mathrm{g} / \mathrm{ml}, 10 \mathrm{~min}$ before ANP) blocked the effects of ANP on MLC phosphorylation (Fig. 4A) and stress fiber formation (Fig. 4C). The NPR-C agonist cANF ( $1 \mu \mathrm{M}, 30$ min) was unable to show beneficial effects (Fig. 4, A and C). Thus, NPR-A/B could be regarded as the major receptors for transducing the actions of ANP in our setting.

\section{Discussion}

Many severe pathologic conditions, such as sepsis or atherosclerosis, are associated with an inflammation-impaired endothelial barrier function leading to an increased plasma extravasation and thus edema formation (Volk and Kox, 2000; Poredos, 2001). Proinflammatory mediators, such as TNF- $\alpha$ or histamine, are involved in the pathogenesis of these disorders and are strong inducers of vascular leakage.
Current therapies against an inflammation-evoked barrier dysfunction (e.g., the administration of glucocorticoids or antihistamines) are often insufficient or even fail (van Nieuw Amerongen and van Hinsbergh, 2002). Therefore, new therapeutical options are needed. Strong progress has been made in recent years concerning the mechanisms involved in the regulation of endothelial permeability (Mehta and Malik, 2006). However, substances that counteract an inflammation-induced vascular leakage by specifically influencing these mechanisms are still largely lacking (van Nieuw Amerongen and van Hinsbergh, 2002).

Initially, the physiological action of the cardiovascular hormone ANP (i.e., the reduction of blood pressure) was mainly ascribed to its natriuretic, diuretic, and vasodilating action. However, ANP was also found to increase endothelial permeability (Huxley et al., 1987). This effect was proven to be
A

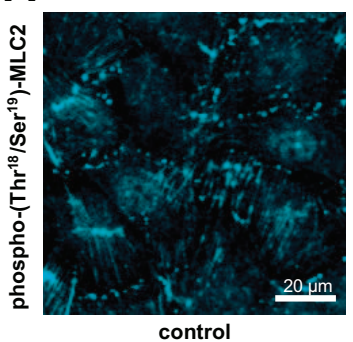

control

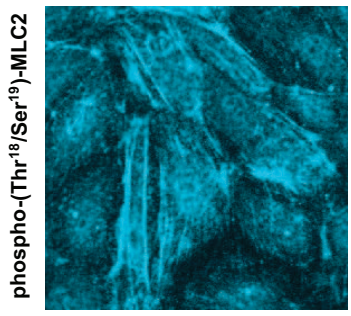

HS-142-1 + ANP + histamine

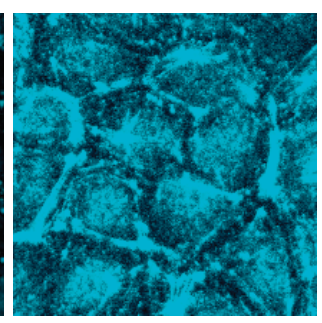

histamine

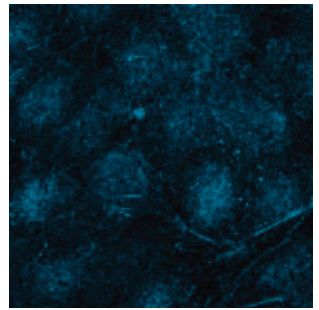

HS-142-1

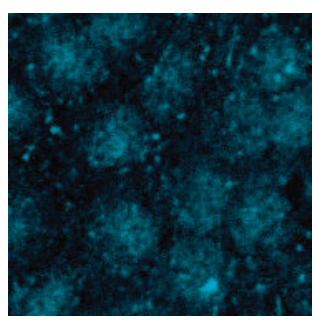

ANP + histamine

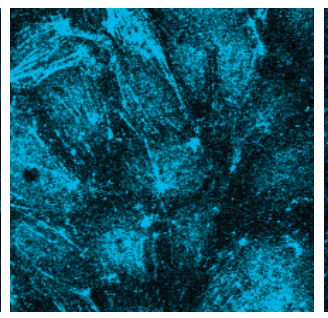

cANF + histamine

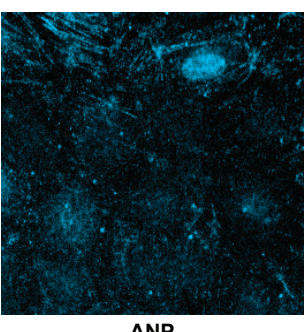

ANP

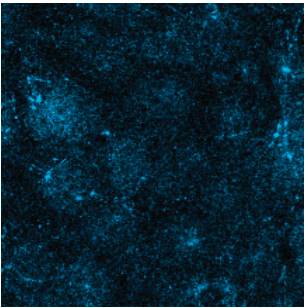

cANF

B

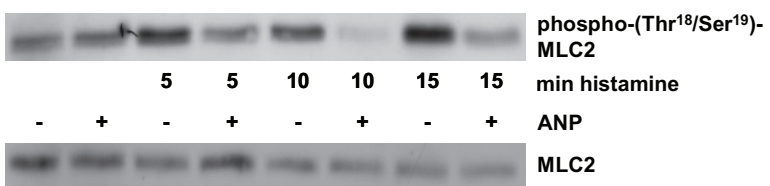

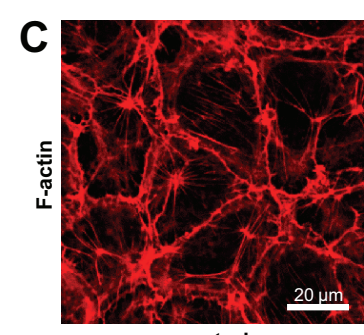

control

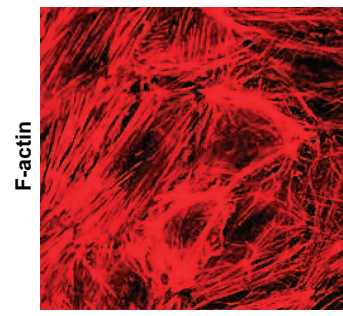

HS-142-1 + ANP + histamine

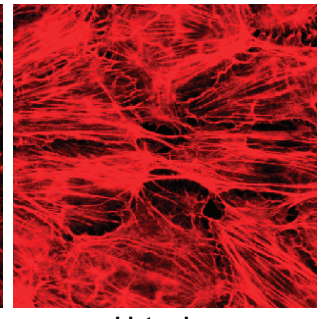

histamine

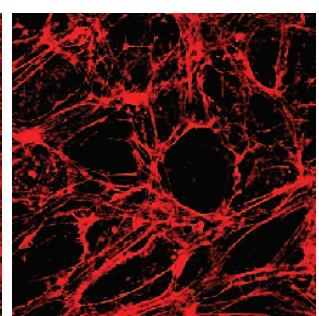

HS-142-1

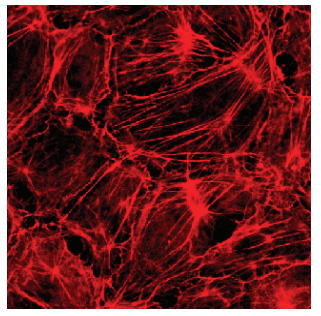

ANP + histamine

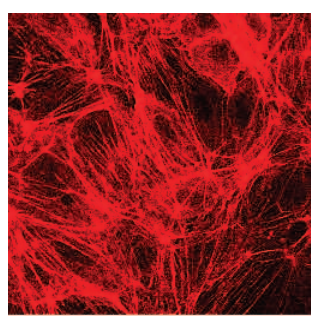

cANF + histamine

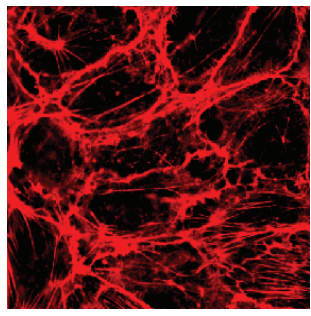

ANP

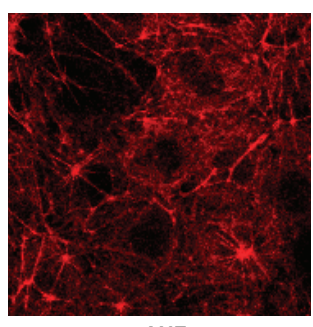

Fig. 4. ANP inhibited histamine-induced MLC2 $\mathrm{Thr}^{18} / \mathrm{Ser}^{19}$-phosphorylation and stress fiber formation. A-C, HUVECs were left untreated (control) or were treated with histamine $(1 \mu \mathrm{M}$, $5 \mathrm{~min}$ ) alone or in combination with ANP (1 $\mu \mathrm{M}, 30 \mathrm{~min}$ pretreatment) or the NPR-C agonist cANF $(1 \mu \mathrm{M}, 30$ min pretreatment). The NPR-A/B receptor antagonist HS142-1 (10 $\mu \mathrm{g} / \mathrm{ml})$ was given $10 \mathrm{~min}$ before ANP. MLC2 $\mathrm{Thr}^{18} / \mathrm{Ser}^{19}$-phosphorylation and $\mathrm{F}$-actin were analyzed via immunocytochemistry and confocal fluorescence microscopy (A and C). MLC2 $\mathrm{Thr}^{18}$ / Ser ${ }^{19}$-phosphorylation was additionally analyzed via Western blot (B). One representative image of at least three independent experiments is shown. 
crucial for the long-term control of plasma volume by ANP (Sabrane et al., 2005). Beyond these permeability-increasing effects on quiescent endothelial cells, ANP has increasingly been recognized to possess barrier-protecting actions on an inflammation-activated endothelium. We could demonstrate that ANP attenuates the TNF- $\alpha$-induced expression of adhesion molecules and monocyte chemoattractant protein-1 by inhibiting NF- $\kappa \mathrm{B}$ activation and p38 mitogen-activated protein kinase signaling (Kiemer et al., 2002b; Weber et al., 2003). In this context, we showed that ANP protects against TNF- $\alpha$-evoked endothelial barrier dysfunction in HUVECs (Kiemer et al., 2002a). ANP was also shown to lower endothelial leakage in vitro induced by the pro-inflammatory stimuli thrombin (Baron et al., 1989) and VEGF (Pedram et al., 2002).

Thus, ANP has commonly been suggested to work as a barrier-protecting agent. Surprisingly, an obvious question remains unanswered precisely: can ANP be used as pharmacological agent to prevent endothelial barrier dysfunction in vivo? This issue is of special interest, because the drug ANP (carperitide) could open new therapeutical options for protecting endothelial barrier function. In the present study, we for the first time show that ANP administered at a pharmacological concentration is able to prevent endothelial leakage in a (histamine-induced) inflammatory setting in vivo. Different aspects of endothelial permeability were used as readout parameters and were all beneficially influenced by ANP: macromolecular permeability (FITC-dextran extravasation), plasma volume/fluid changes (hematocrit), and electrical resistance (TEER measurement). Compared with the maximal increase of FITC-dextran extravasation induced by histamine (time point 45 min in Fig. 1A), ANP led to a reduction of approximately $65 \%$. Given this pronounced effect, a therapeutical impact of ANP is not unlikely. A complete blockage of the deleterious effect of histamine was observed in the presence of an extraordinarily high dosage of the glucocorticoid dexamethasone, a highly potent anti-inflammatory drug.

Former studies dealing with ANP and vascular permeability served as valuable hints toward an in vivo relevance of ANP as barrier-protecting agent. However, these reports did not concisely test the hypothesis that administered ANP exerts beneficial effects on endothelial barrier dysfunction in vivo, because they either used ex vivo models or focused on the endogenous ANP system. 1) Three older reports demonstrate that pharmacological concentrations of ANP attenuate changes of pulmonary wet weight induced by toxic agents, such as reactive oxygen metabolites, paraquat, or arachidonic acid in ex vivo models of isolated-perfused lungs from rabbits or guinea pigs (Inomata et al., 1987; Imamura et al., 1988; Lofton et al., 1991). 2) Blockade of endogenous ANP was shown to deteriorate pulmonary edema formation in rats suffering from high-altitude-induced (Irwin et al., 2001) or HCl-evoked (Wakabayashi et al., 1990) pulmonary vascular leakage, whereas mice lacking the major ANP-degrading enzyme neutral endopeptidase were found to be less susceptible for pulmonary leakage (Irwin et al., 2005a). It is noteworthy that Pedram et al. (2002) showed that VEGF-induced vascular leakage is attenuated in ANP-overexpressing mice, whereas these mice are not protected against histamineevoked leakage. This might be due to the much lower ANP levels in these animals (plasma level: $\sim 40 \mathrm{pM}$ ) compared with our setting, in which ANP is exogenously supplied to reach a pharmacological plasma concentration of $200 \mathrm{nM}$. Recently, our group could demonstrate that ANP-treated mice (plasma level, $\sim 35 \mathrm{nM}$ ) are protected against LPSinduced septic shock (Ladetzki-Baehs et al., 2007). Because endothelial hyperpermeability is an important pathological feature of sepsis, it can be speculated that the barrier-protecting effect of ANP contributes to the beneficial action in the mouse septic shock model. Our results suggest that pharmacological concentrations of ANP show additional, highly valuable effects beyond its action as an endogenous regulator of permeability.

Adherens junctions and the contractile apparatus are key players in the regulation of endothelial permeability. Both the loss of VE-cadherin function and the activation of MLC result in decreased transendothelial electrical resistance (Garcia et al., 1997; van Buul et al., 2005) and increased macromolecular permeability (Garcia et al., 1995; Nwariaku et al., 2002). Studies investigating the action of ANP on these key systems are as yet completely lacking. We provide the first evidence that ANP interacts with these systems, because we showed that ANP attenuated both adherens junction disassembly (morphological changes and $\mathrm{Tyr}^{731}$ phosphorylation of VE-cadherin) and activation of the contractile apparatus (phosphorylation of MLC and rearrangement of F-actin) induced by histamine. Furthermore, we could demonstrate that ANP exerts these effects predominantly via NPR-A. Because this receptor represents particulate guanylate cyclases, it can be speculated that the actions of ANP might be mediated via the second messenger cyclic GMP. Our results add further support to the hypothesis that ANP is an endothelium-protecting agent, because it directly counteracts the detrimental effects of proinflammatory mediators on endothelial barrier function.

Few data exist regarding the action of ANP on subcellular systems and its contribution to permeability regulation. We and others could demonstrate that ANP inhibits F-actin stress fiber formation induced by TNF- $\alpha$ (Kiemer et al., 2002a; Irwin et al., 2005b) or VEGF (Pedram et al., 2002). Interestingly, one study reports that ANP influences tight junctions in bovine aortic endothelial cells (Pedram et al., 2002). In contrast to the dense aortic endothelium, the occurrence of tight junctions is limited in the venous endothelium (Ogunrinade et al., 2002), which represents the predominant site of endothelial hyperpermeability and was investigated in the present study.

In summary, we have revealed ANP as a potent endothelial barrier-protecting agent in vivo. Moreover, we have identified adherens junctions and the contractile apparatus as important subcellular systems targeted by ANP. Most importantly, our study highlights ANP as an interesting pharmacological compound opening a new therapeutic option for the prevention of vascular leakage. This warrants further efforts aiming for an expansion of the therapeutic indications of natriuretic peptides.

\section{References}

Baez S (1973) An open cremaster muscle preparation for the study of blood vessels by in vivo microscopy. Microvasc Res 5:384-394.

Baron DA, Lofton CE, Newman WH, and Currie MG (1989) Atriopeptin inhibition of thrombin-mediated changes in the morphology and permeability of endothelial monolayers. Proc Natl Acad Sci U S A 86:3394-3398.

Fürst R, Brueckl C, Kuebler WM, Zahler S, Krötz F, Görlach A, Vollmar AM, and Kiemer AK (2005) Atrial natriuretic peptide induces mitogen-activated protein 
kinase phosphatase-1 in human endothelial cells via Rac1 and NAD(P)H oxidase/ Nox2-activation. Circ Res 96:43-53.

Garcia JG, Davis HW, and Patterson CE (1995) Regulation of endothelial cell gap formation and barrier dysfunction: role of myosin light chain phosphorylation. J Cell Physiol 163:510-522.

Garcia JG, Schaphorst KL, Shi S, Verin AD, Hart CM, Callahan KS, and Patterson CE (1997) Mechanisms of ionomycin-induced endothelial cell barrier dysfunction. Am J Physiol 273:L172-L184.

Hatakeyama T, Pappas PJ, Hobson RW, Boric MP, Sessa WC, and Duran WN (2006) Endothelial nitric oxide synthase regulates microvascular hyperpermeability in vivo. J Physiol 574:275-281.

Huxley VH, Tucker VL, Verburg KM, and Freeman RH (1987) Increased capillary hydraulic conductivity induced by atrial natriuretic peptide. Circ Res 60:304-307. Imamura T, Ohnuma N, Iwasa F, Furuya M, Hayashi Y, Inomata N, Ishihara T, and Noguchi T (1988) Protective effect of alpha-human atrial natriuretic polypeptide (alpha-HANP) on chemical-induced pulmonary edema. Life Sci 42:403-414

Inomata N, Ohnuma N, Furuya M, Hayashi Y, Kanai Y, Ishihara T, Noguchi T, and Matsuo H (1987) Alpha-human atrial natriuretic peptide (alpha-HANP) prevents pulmonary edema induced by arachidonic acid treatment in isolated perfused lung from guinea pig. Jpn $J$ Pharmacol 44:211-214.

Irwin DC, Patot MT, Tucker A, and Bowen R (2005a) Neutral endopeptidase null mice are less susceptible to high altitude-induced pulmonary vascular leak. High Alt Med Biol 6:311-319.

Irwin DC, Rhodes J, Baker DC, Nelson SE, and Tucker A (2001) Atrial natriuretic peptide blockade exacerbates high altitude pulmonary edema in endotoxin-primed rats. High Alt Med Biol 2:349-360.

Irwin DC, Tissot van Patot MC, Tucker A, and Bowen R (2005b) Direct ANP inhibition of hypoxia-induced inflammatory pathways in pulmonary microvascular and macrovascular endothelial monolayers. Am J Physiol Lung Cell Mol Physiol 288:L849-L859.

Kiemer AK, Fürst R, and Vollmar AM (2005) Vasoprotective actions of the atrial natriuretic peptide. Curr Med Chem Cardiovasc Hematol Agents 3:11-21.

Kiemer AK and Vollmar AM (2001) The atrial natriuretic peptide regulates the production of inflammatory mediators in macrophages. Ann Rheum Dis 60 Suppl 3:iii68-iii70.

Kiemer AK, Weber NC, Fürst R, Bildner N, Kulhanek-Heinze S, and Vollmar AM (2002a) Inhibition of P38 MAPK activation via induction of MKP-1: atrial natriuretic peptide reduces TNF- $\alpha$-induced actin polymerization and endothelial permeability. Circ Res 90:874-881.

Kiemer AK, Weber NC, and Vollmar AM (2002b) Induction of $\mathrm{I}_{\kappa} \mathrm{B}$ : atrial natriuretic peptide as a regulator of the NF-Kb pathway. Biochem Biophys Res Commun 295:1068-1076.

Ladetzki-Baehs K, Keller M, Kiemer AK, Koch E, Zahler S, Wendel A, and Vollmar AM (2007) Atrial natriuretic peptide, a regulator of nuclear factor-kappab activation in vivo. Endocrinology 148:332-336.
Lofton CE, Baron DA, Heffner JE, Currie MG, and Newman WH (1991) Atrial natriuretic peptide inhibits oxidant-induced increases in endothelial permeability. J Mol Cell Cardiol 23:919-927.

Mehta D and Malik AB (2006) Signaling mechanisms regulating endothelial permeability. Physiol Rev 86:279-367.

Morishita Y, Sano T, Ando K, Saitoh Y, Kase H, Yamada K, and Matsuda Y (1991) Microbial polysaccharide, HS-142-1, competitively and selectively inhibits ANP binding to its guanylyl cyclase-containing receptor. Biochem Biophys Res Commun 176:949-957.

Nwariaku FE, Liu Z, Zhu X, Turnage RH, Sarosi GA, and Terada LS (2002) Tyrosine phosphorylation of vascular endothelial cadherin and the regulation of microvascular permeability. Surgery 132:180-185.

Ogunrinade O, Kameya GT, and Truskey GA (2002) Effect of fluid shear stress on the permeability of the arterial endothelium. Ann Biomed Eng 30:430-446.

Pedram A, Razandi M, and Levin ER (2002) Deciphering vascular endothelial cell growth factor/vascular permeability factor signaling to vascular permeability. Inhibition by atrial natriuretic peptide. J Biol Chem 277:44385-44398.

Poredos P (2001) Endothelial dysfunction in the pathogenesis of atherosclerosis. Clin Appl Thromb Hemost 7:276-280.

Potter MD, Barbero S, and Cheresh DA (2005) Tyrosine phosphorylation of VEcadherin prevents binding of P120- and beta-catenin and maintains the cellular mesenchymal state. J Biol Chem 280:31906-31912.

Sabrane K, Kruse MN, Fabritz L, Zetsche B, Mitko D, Skryabin BV, Zwiener M, Baba HA, Yanagisawa M, and Kuhn M (2005) Vascular endothelium is critically involved in the hypotensive and hypovolemic actions of atrial natriuretic peptide. $J$ Clin Invest 115:1666-1674.

van Buul JD, Anthony EC, Fernandez-Borja M, Burridge K, and Hordijk PL (2005) Proline-rich tyrosine kinase 2 (Pyk2) mediates vascular endothelial-cadherinbased cell-cell adhesion by regulating beta-catenin tyrosine phosphorylation. J Biol Chem 280:21129-21136.

van Nieuw Amerongen GP, and van Hinsbergh VW (2002) Targets for pharmacological intervention of endothelial hyperpermeability and barrier function. Vascu Pharmacol 39:257-272.

Volk T and Kox WJ (2000) Endothelium function in sepsis. Inflamm Res 49:185-198. Wakabayashi G, Ueda M, Aikawa N, Naruse M, and Abe O (1990) Release of ANP and its physiological role in pulmonary injury due to HCl. Am J Physiol 258:R690 R696.

Weber NC, Blumenthal SB, Hartung T, Vollmar AM, and Kiemer AK (2003) ANP inhibits TNF-alpha-induced endothelial MCP-1 expression - involvement of p38 MAPK and MKP-1. J Leukoc Biol 74:932-941.

Address correspondence to: Dr. Robert Fürst, Department of Pharmacy, Pharmaceutical Biology, University of Munich, Butenandtstr. 5-13, 81377 Munich, Germany. E-mail: robert.fuerst@cup.uni-muenchen.de 